\title{
Sonographically detected costo-chondral dislocation in an abused child
}

\section{A new sonographic sign to the radiological spectrum of child abuse}

\author{
A.J.Smeets, S. G.F. Robben and M. Meradji \\ Department of Radiology, Sophia Children's Hospital, Erasmus University Rotterdam, Rotterdam, The Netherlands
}

Received: 2 February 1990; accepted: 29 March 1990

\begin{abstract}
A case of an abused child with fractures of the skull, ribs and long bones is presented. Sonographically a costochondral dislocation of the left lower ribs was detected, which has not been reported in the literature.
\end{abstract}

When Caffey introduced the term "intentional ill-treatment" in 1946 describing six young patients with subdural hematomas and coexisting fractures in the long bones without a history of trauma, he laid the basis of what later developed into the battered child syndrome.

The characteristics of this syndrome are surface marks (bruising, abrasions, burns), skeletal injuries (metaphyseal-, epiphyseal-, skull-and rib fractures), visceral injuries, subdural hematomas and ocular injuries. Beside these hallmarks there remains a large variety of possible signs such as costo-chondral dislocation as presented in this case.

\section{Case report}

A nine-month-old girl was admitted to the First Aid department of our hospital. Two days previous to the hospital admittance she had fallen from the bench. A progressive swelling of the left flank resulted.

The physical examination showed an active healthy child, who had small wounds on her left foot and left elbow. The thorax and $a b-$ domen were swollen and tender on the left side. There were no further abnormalities.

The standard AP X-thorax showed healing fractures of the posterior ribs 7 through 10 on the left side, a healing fracture of 8 th rib and a fresh fracture of the 11 th rib on the right posterior side. Moreover there was a pleural effusion and a soft tissue swelling on the left lat- eral thoracic wall (Fig. 1). The plain abdominal radiograph showed no specific findings. Additional radiographs were taken to assess the diagnosis battered child. The skull x-ray showed a fracture in the occipital bone and there were metaphyseal/epiphyseal fractures in both distal femora, proximal tibiae (Fig. 2) and proximal left humerus. No signs of generalised bone disease or bone dysplasia were present.

Sonography of the skull and upper abdomen revealed no signs of intracerebral or subdural hematoma or rupture of upper abdominal organs. However at the site of the left flank mass there was a large subcutaneous hematoma near the ribs and a costo-chondral dislocation of all left lower ribs. The chondral parts of the ribs had been dislocated inwards for $1 \mathrm{~cm}$ (Fig. 3). During respiratory movements it was clearly visible that the bony and chondral parts of the ribs were no longer attached to each other. In addition consolidated lung parenchyma accompanied by pleural effusion, probably due to lung contusion, was seen.

During hospital admittance she recovered well. The diagnosis battered child syndrome was confirmed and the parents were relieved from parental custody.

\section{Discussion}

Multiple fractures in a child should raise the suspicion of child abuse especially if there is no history of adequate trauma. If the fractures are old, multiple, metaphyseal/epiphyseal or situated in the ribs the diagnosis is almost certain [1]. Rib fractures are specific for physical abuse in young children (especially those under one year old). The only other cause for rib fractures in this age group is generalised bone disease or dysplasia [2]. Rib fractures are often situated on the posterior arc of the rib near the costotransverse articulation. Usually there is no displacement or angulation of these posterior rib fractures. As a result of this location and the lack of dislocation fresh fractures are hard to see on standard AP radiographs. An oblique radiograph will often be very helpful [3].
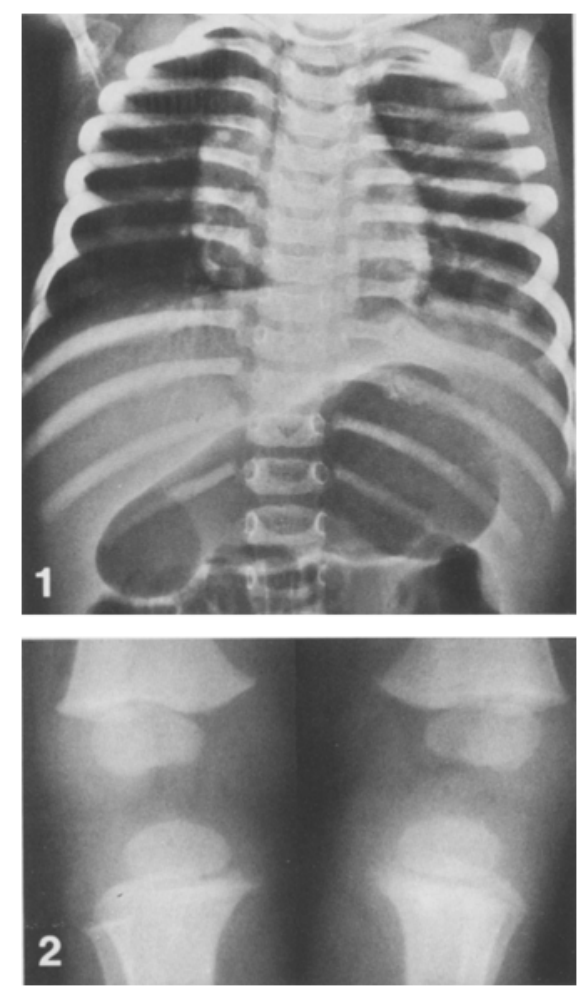

Fig. 1. Standard AP chest X-ray. Healing and fresh rib fractures, pleural effusion and soft tissue swelling

Fig.2. Metaphyseal/epiphyseal fractures in distal femora and proximal 

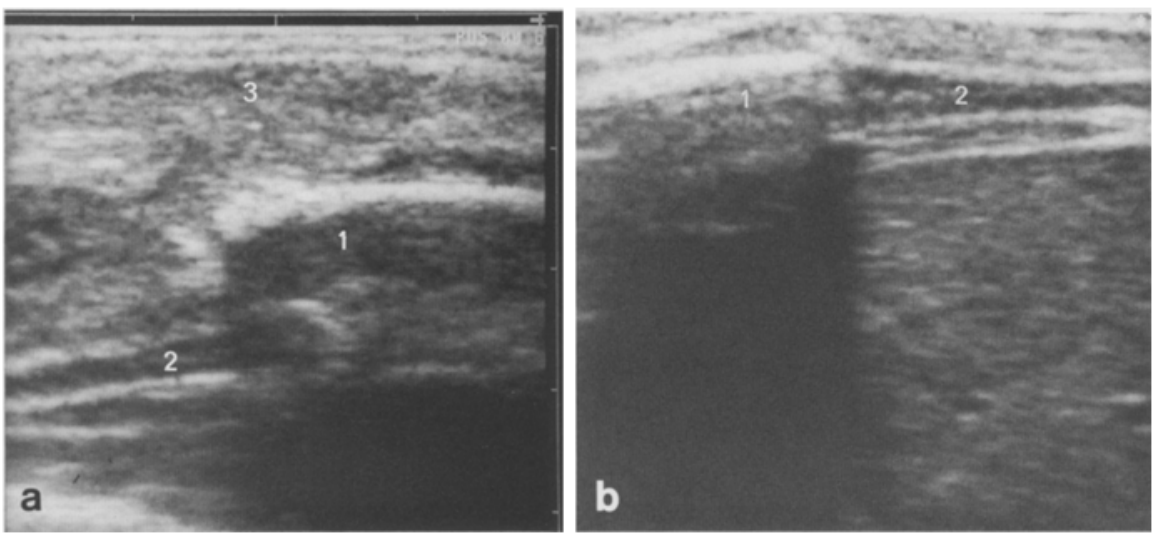

Fig. 3a, b. Sonograph of costo-chondral dislocation (a) and a normal costo-chondral junction for comparison (b). 1 = bony part of rib; 2 = chondral part of rib; 3 = subcutaneous hematoma

Healing fractures are easier to identify because of the often luxurious callus formation. Rib fractures are often bilateral and multiple [4]. The most common mechanism to induce these rib fractures is $\mathrm{AP}$ compression with the abuser's palms held laterally on the thorax, the thumbs anteriorly and the fingers posteriorly during violent shaking in AP direction.

In this case the possible AP compression had not only resulted in several pos- terior arc fractures but also in a costochondral dislocation in the left lower ribs. Because the chondral parts of the ribs are not visible on $\mathrm{X}$-rays this dislocation is impossible to see until in the healing phase, even then this diagnosis is difficult - especially the differentiation with rickets [3]. Because of the difficulty in diagnosing costo-chondral dislocation and the lack of signs on the standard radiographs we suspect that this diagnosis is often missed. If there is reason to think of this kind of injury - as in this case with bruising, hematoma and pleural effusion - sonography will easily reveal this injury and is a simple extension of the abdominal sonography which to our opinion should be routinely performed in an abused child with lower rib fractures to exclude abdominal organ injury.

\section{Literature}

1. Hobbs CJ (1989) ABC of child abuse. Fractures. BMJ 298: 1015

2. Feldman KW, Brewer DK (1984) Child abuse, cardiopulmonary resuscitation, and rib fractures. Pediatrics 73: 339

3. Cameron, Rea (1975) Atlas of the battered child syndrome. Churchill Livingston, Edinburgh London New York

4. Kleinman PK (1987) Diagnosticimaging of child abuse, 1stedn. Williams and Wilkings, Baltimore London Los Angeles

Dr. A.J.Smeets

Department of Radiology

Dijkzigt Hospital

Dr. Molewaterplein 40

NL-3015 GD Rotterdam

The Netherlands 